\title{
Mean-field analysis of the stability of a K-Rb Fermi-Bose mixture
}

\author{
M. Modugno, ${ }^{1,2}$ F. Ferlaino, ${ }^{1}$ F. Riboli, ${ }^{1, *}$ G. Roati, ${ }^{1}$ G. Modugno, ${ }^{1}$ and M. Inguscio ${ }^{1}$ \\ ${ }^{1}$ LENS - Dipartimento di Fisica, Università di Firenze and INFM \\ Via Nello Carrara 1, 50019 Sesto Fiorentino, Italy \\ ${ }^{2}$ BEC-INFM Center, Università di Trento, 38050 Povo, Italy
}

(Dated: November 13, 2018)

\begin{abstract}
We compare the experimental stability diagram of a Fermi-Bose mixture of ${ }^{40} \mathrm{~K}$ and ${ }^{87} \mathrm{Rb}$ atoms with attractive interaction to the predictions of a mean-field theoretical model. We discuss how this comparison can be used to give a better estimate of the interspecies scattering length, which is currently known from collisional measurements with larger uncertainty.
\end{abstract}

PACS numbers: 03.75.Ss, 05.30.Hh, 32.80.Pj

The production of degenerate Fermi-Bose mixtures of atoms [1, 2, 3, [4] has opened new directions in the field of ultracold atomic gases, with relevant implications for the achievement of fermionic superfluidity. Collisional interactions between bosons and fermions have a twofold role: they allow for an efficient sympathetic cooling of the fermions into quantum degeneracy, and they affect the properties of the degenerate mixture. In case of a large boson-fermion attraction, the most important predicted effects are mean-field instabilities [5, 6, 7] and boson-induced interactions between fermions [8], besides a general modification of the properties of the two individual components $9,10,11$. We have recently observed the collapse of the Fermi gas in a ${ }^{40} \mathrm{~K}-{ }^{87} \mathrm{Rb}$ mixture [12], which is indeed characterized by a large attractive interaction between the components.

In our study of the collapse we found that the order of magnitude for the critical atom numbers is in agreement with the predictions of a mean-field model for spherical geometry [6].

In this work we investigate the stability diagram of the K-Rb system, and we make a quantitative comparison of the experimental findings with mean-field theory taking into account the actual trapping potential for the atoms. We find that the mean-field model is able to reproduce the critical atom numbers for collapse using a value for the boson-fermion scattering length $a_{B F}$ that is in good agreement with the one we determined through the study of cold collisions [13]. Moreover, we show how the comparison of theory and experiment on the stability diagram can be used to determine the scattering length with a much lower uncertainty than that determined from collisional measurements.

The Fermi-Bose mixtures of ${ }^{40} \mathrm{~K}$ (fermions) and ${ }^{87} \mathrm{Rb}$ (bosons) atoms are produced by sympathetic cooling in a magnetic potential 4]. Both species are trapped in their low-field-seeking states with largest magnetic moment,

\footnotetext{
*Present address: INFM-Dipartimento di Fisica, Università di
} Trento, Via Sommarive 14, 38050 Povo, Italy which experience potentials of the kind

$$
\begin{aligned}
& V_{B}(\mathbf{x})=\frac{1}{2} m_{B}\left[\omega_{B \perp}^{2}\left(x^{2}+y^{2}\right)+\omega_{B z}^{2} z^{2}\right] \\
& V_{F}(\mathbf{x})=\frac{1}{2} m_{F}\left[\omega_{F \perp}^{2}\left(x^{2}+\left(y-y_{0}\right)^{2}\right)+\omega_{F z}^{2}\left(z-z_{0}\right)^{2}\right] .
\end{aligned}
$$

The trap frequencies are $\omega_{F \perp}=2 \pi \times 317 \mathrm{~Hz}, \omega_{F z}=$ $2 \pi \times 24 \mathrm{~Hz}$ for ${ }^{40} \mathrm{~K}$, while those of ${ }^{87} \mathrm{Rb}$ are a factor $\sqrt{m_{B} / m_{F}} \simeq 1.47$ smaller. The system is characterized by a gravitational sag between the two clouds, which affects both the horizontal and vertical directions, due to a small misalignment of the magnetic trap with respect to the direction of gravity [14]. The values of the displacement between the two potential centers are $y_{0}=3.6 \mu \mathrm{m}$ and $z_{0}=20 \mu \mathrm{m}$.

The largest samples that we are able to cool to quantum degeneracy amount to about $2 \times 10^{5}$ bosons coexisting with $5 \times 10^{4}$ fermions. The atom numbers for both species can be adjusted separately by controlling the loading and the evaporation procedures. The coldest Fermi gas observed have a temperature of about $0.2 T_{F}$, and the minimum temperature of the bosons that we are able to measure from the non-condensed fraction is limited to about $0.6 T_{c}$.

In the mean-field model that we use to describe this Fermi-Bose system, the ground state is determined by the solution of Gross-Pitaevskii (GP) equation for the bosons, coupled to the Thomas-Fermi equation for the fermions $[\underline{5}, \underline{6}]$

$$
\begin{aligned}
& {\left[-\frac{\hbar^{2}}{2 m_{B}} \nabla^{2}+V_{B}+g_{B B} n_{B}+g_{B F} n_{F}\right] \phi=\mu_{B} \phi} \\
& n_{F}={\frac{\left(2 m_{F}\right)^{3 / 2}}{6 \pi^{2} \hbar^{3}}}^{\left(\epsilon_{F}-V_{F}-g_{B F} n_{B}\right)^{3 / 2}}
\end{aligned}
$$

where $n_{B}=\left|\phi^{2}\right|$ and $n_{F}$ are the boson and fermion densities respectively, and $\phi(\mathbf{x})$ is the condensate wave function. The boson-boson and boson-fermion interactions are described by the coupling constants $g_{B B}=$ $4 \pi \hbar^{2} a_{B B} / m_{B}$ and $g_{B F}=2 \pi \hbar^{2} a_{B F} / m_{R}$ in terms of the $s$-wave scattering length $a_{B B}$ and $a_{B F}$. Here $m_{B, F}$ are the atomic masses and $m_{R}=m_{B} m_{F} /\left(m_{B}+m_{F}\right)$ is the reduced mass. 
In the case of the rubidium-potassium mixture, the best experimental estimates of the scattering lengths are $a_{B B}=98.98 \pm 0.02 a_{0}[15]$ and $a_{B F}=-410 \pm 80 a_{0}[13$. The difference in the uncertainties for the two quantities stems from the different techniques used, which is a combination of photoassociation and Feshbach spectroscopies in the first case, and measurement of cold collisions in the second case. Although the investigation of cold collisions is the easiest way to measure the interactions in an ultracold gas, it is often accompanied by a rather large uncertainty. Indeed, what one measures in the experiment is a collisional rate, which at ultralow temperature has a dependence

$$
\Gamma \propto N a^{2}
$$

on the atom number $N$ and on the scattering length $a$. The absolute atom number, in the case of a mixture of two species, cannot be determined with an accuracy typically better that $40 \%$, giving rise to a $20 \%$ uncertainty on the scattering length.

By solving equations (21)-(3) we can investigate the ground state and the stability of the system against collapse. The equations are solved with an imaginary-time evolution, embedded in an iterative scheme, as in [6]. The stability is checked by requiring that energies, chemical potentials and central densities converge to the final value, with a precision at least of $10^{-6}$. In Fig. 1 we show a typical ground state configuration, calculated with the nominal values $a_{B F}=-410 a_{0}, N_{B}=7.5 \cdot 10^{4}, N_{F}=2 \cdot 10^{4}$. As already discussed in Refs. [5, 6], the mutual attraction results in an enhancement of the density of both species in the volume of overlap. We note that the gravitational sag, that shifts the centers of the two clouds, reduces the effect of the mutual attraction with respect to the concentric case studied in Ref. [6].

The deformed ground state of the system is predicted to lead to a series of effects that can be described within a mean-field approach, including a modification of the frequencies of collective excitations 9,11 and of the expansion of the two clouds from the trap [10]. We actually observed the latter effect already from the first experiments [4], where we had an evidence of a modified expansion of the condensate in presence of the Fermi gas.

If the atom numbers are increased above some critical values, an instability occurs. In our model, accordingly to the study reported in Refs. [6, 16] the signature of the instability is the failure of the convergence procedure during the iterative evolution toward the ground state of the system. In particular, the onset of instability is characterized by an indefinite growth of central densities which triggers the simultaneous collapse of the two species. We note that the investigation of the actual dynamics after the system has been driven into the unstable region would require a description that goes beyond Eqs. (2)-(3), in a similar fashion to what happens during the collapse of a single Bose-Einstein condensate with attractive interaction [17, 18]. Here we will not discuss these aspects, since we are concerned with the determination
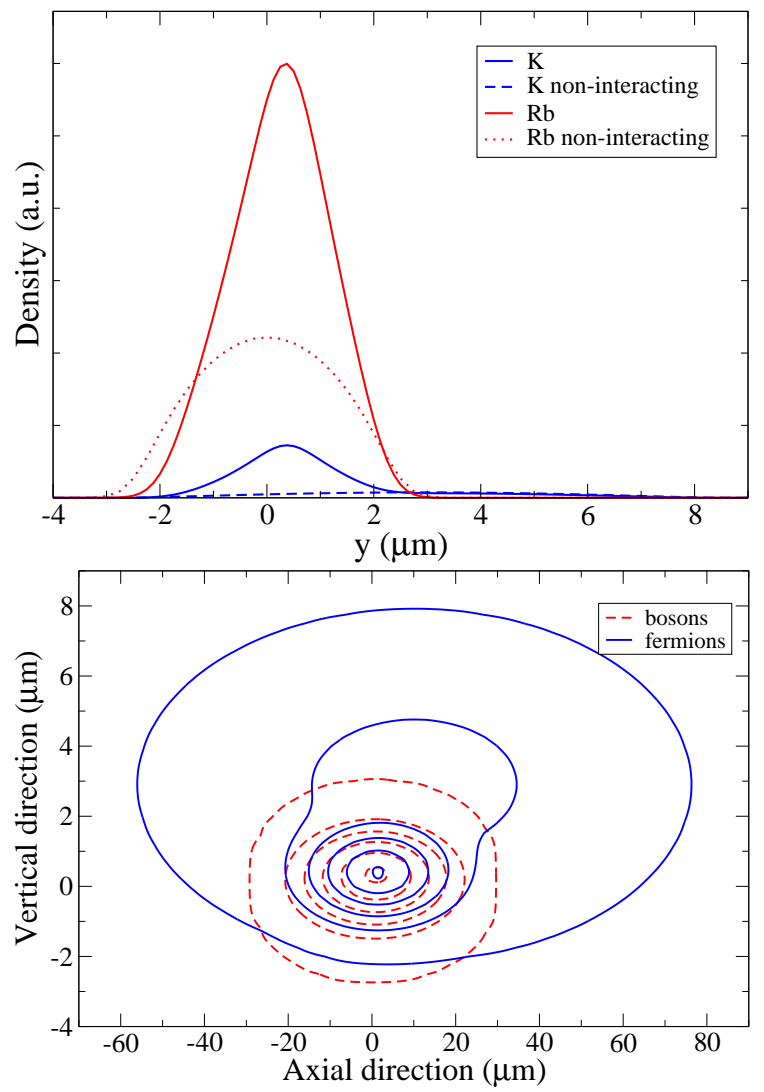

FIG. 1: Density profiles along the vertical $y$ direction (a) and contour levels in the $(y-z)$ plane (b) of the ground-state of the Fermi-Bose mixture, for $a_{B F}=-410 a_{0}, N_{B}=7.5 \cdot 10^{4}$, $N_{F}=2 \cdot 10^{4}$.

of the critical values for the onset of instabilities only.

In the experiment we observe the instability of the Fermi-Bose system as the number of condensed bosons in increased by evaporation, at a fixed number of fermions 12]. The signature of the instability is a sudden loss of most of the fermions from the magnetic trap, which we attribute to a largely increased three-body recombination in the collapsing samples. Here we are interested just in the critical atom numbers, but we note that further experiments, for example using a Feshbach resonance to tune $a_{B F}$ [13], will be necessary to study the collapse dynamics of the system.

To compare the predictions of the mean-field model to the experimental findings on the instability, we have built a stability diagram, shown in Fig. 2] Here we report in the plane $N_{B}-N_{F}$ the condensate and fermion atom numbers that we were able to measure in the experiment for stable samples, and compare them with the calculated critical line for instability. We note that all the data points refer to samples at temperatures of the order of $0.2-0.5 T_{F}$ for the Fermi gas, and with almost no detectable thermal fraction for the BEC. In the model, the occurrence of instability depends on both $N_{B}, N_{F}$, and also on the value of the scattering length $a_{B F}$. In 


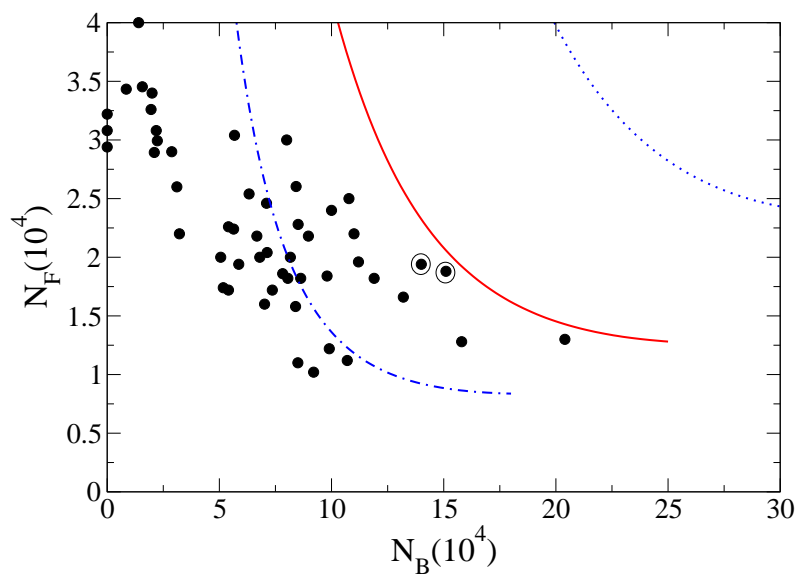

FIG. 2: Region of stability of the Fermi-Bose mixture, as a function of the number of atoms. The black dots are the experimental points; lines are the theoretical prediction for the boundary between the stable (left) and collapse (right) regions, for three values of the inter-particle scattering length (in units of the Bohr radius $\left.a_{0}\right): a_{B F}=-380 a_{0}$ (dotted line), $a_{B F}=-395 a_{0}$ (continuous line), $a_{B F}=-410 a_{0}$ (dasheddotted line). The marked dots are found very close to the instability (see text).

Fig. 2 we have therefore plotted a family of critical lines by varying $a_{B F}$ around the nominal value. Note that the position of the critical line depends quite strongly on the value of $a_{B F}$, and it shifts toward larger atom numbers for decreasing magnitudes of $a_{B F}$. Although the critical line calculated for the nominal value $a_{B F}=-410 a_{0}$ is in rather good agreement with the experimental observation, the one calculated for $a_{B F}=-395 a_{0}$ better fits the data. In the experiment we observed the collapse of the Fermi gas for number pairs close to the two marked data points [19], which appears to be actually the region of achievable number pairs closest to the instability.

The strong dependence on the criticality on $a_{B F}$ that we find in the mean-field analysis is in accordance with the scaling law for the critical number of condensate atoms

$$
N_{B}^{c r i t} \sim\left|a_{B F}\right|^{-\alpha}
$$

with $\alpha=12$, discussed in 5, 7]. Here we have verified that this scaling behavior perfectly fits the numerical solutions of Eqs. (2)-(3) in case of a simplified concentric spherical configuration, by using an average trapping frequency as usually found in literature [6, 20], as shown in Fig. 3 In this calculation we have kept the number ratio $N_{B} / N_{F}$ fixed, which corresponds to move along a straight line passing from the origin in the diagram of Fig. 2 This calculation also shows that the 3D geometry of the experiment results in a renormalization of the scaling exponent, $\alpha_{3 D} \simeq 11$, besides an effective increase (in modulus) of the critical scattering length, of the order $2 \%$, due to the presence of gravity. In fact, the effect of the gravitational shift is a reduction of the density

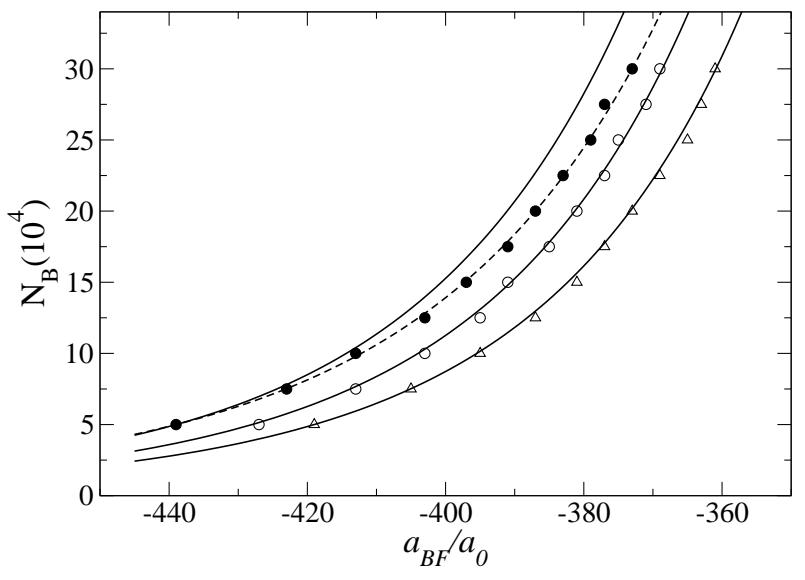

FIG. 3: Behavior of the critical number of atoms $N_{B}$ as a function of the scattering length $a_{B F}$, for $N_{B}=4.25 N_{F}$ (triangles) and $N_{B}=8.5 N_{F}$ (circles). The numerical solutions of the mean-field equations (symbols) are compared with the scaling law in Eq. (5) with exponent $\alpha=12$ (continuous line) and $\alpha=11$ (dashed line). Filled and empty symbols refer respectively to the full 3D geometry and to the simplified spherically symmetric case discussed in the text.

enhancements due to the attractive interaction, with respect to the case in which the to species are trapped by concentric potentials.

This analysis suggests the possibility of using a comparison of the experimental and calculated critical atom numbers to extract a precise information on the value of the scattering length for our K-Rb mixture. To account for the uncertainty in the determination of atom numbers in the experiment, in Fig. 4 we have plotted the same data points of Fig. 22 expanding or compressing both scales by $40 \%$, and compared them with the same family of critical lines.

By taking the critical lines that best fit with the observation in these two limiting cases, we can extract the following mean-field estimate for $a_{B F}$

$$
a_{B F}^{m f} \simeq-395 \pm 15 a_{0} .
$$

Note how the dependence of $a_{B F}$ on the number of atoms of Eq. 5 stronger than that of Eq. 4. results in a 5 -fold smaller uncertainty on the scattering length determined by the stability analysis than that obtained with collisional measurements. We expect this meanfield prediction for $a_{B F}$ in Eq. (6) to be rather robust, since the $40 \%$ experimental uncertainty on the number of atoms should be quite larger that the possible discrepancy between the GP theory and the actual behavior of the system, as recently discussed for the case of a single ${ }^{85} \mathrm{Rb}$ Bose-Einstein condensate with attractive interaction 21, 22, 23].

The assumption of the same scaling for boson and fermion atom numbers due to experimental uncertainty is not unrealistic. Indeed, the possible sources of systematic errors in the atom number calibration are residual magnetic fields, non perfect polarization of the probe 

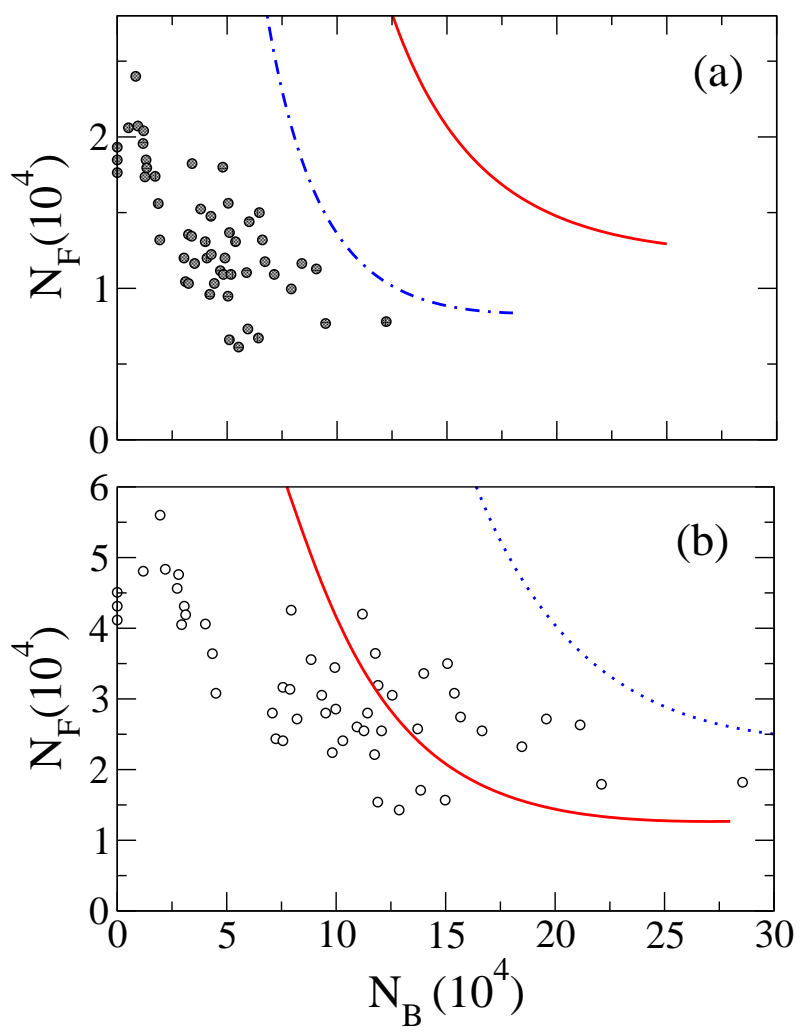

FIG. 4: Region of stability of the Fermi-Bose mixture, as a function of the number of atoms. To keep into account the experimental uncertainty on the number of atoms these have been increased or reduced by a $40 \%$, respectively in (a) and (b). Continuous lines are the theoretical prediction for the boundary between the stable (left) and collapse (right) regions, for three values of the inter-particle scattering length (in units of the Bohr radius $a_{0}$ ): $a_{B F}=-380 a_{0}$ (dotted line), $a_{B F}=-395 a_{0}$ (continuous line), $a_{B F}=-410 a_{0}$ (dasheddotted line). beam, or improper calibration of the imaging magnification. When using a simultaneous imaging scheme [4], all these factors affect in the same way the measured atom numbers for bosons and fermions.

We note that recently it has been proposed a beyond mean-field approach, including the second order correction in the scattering length $a_{B F}$, that should provide important modifications to the mean-field results [20]. Nonetheless we have verified that the inclusion of this term stabilizes the system in the range of atom numbers considered here for any value of the scattering length, thus forbidding the collapse, in contrast with the experimental findings. Higher order terms might therefore be important to provide a correct beyond mean-field description, although at this stage it is not clear whether this could affect the critical number of atoms for collapse.

In conclusion, our mean-field analysis of the stability of the K-Rb Fermi-Bose mixture shows the possibility of an independent determination of the relevant scattering length of the system. The strong dependence of the critical atom numbers on the scattering length allows to largely reduce the effect of the experimental uncertainty. The value we determine for $a_{B F}$ is in very good accordance with that found from collisional measurements. We note that an independent, more precise determination of $a_{B F}$, for example using Feshbach spectroscopy [13], would be useful to test the validity of the mean-field theory for strongly interacting Fermi-Bose mixtures, and to asses the importance of beyond mean-field effects.

\section{Acknowledgments}

This work was supported by MIUR, by EC under contract HPRICT1999-00111, and by INFM, PRA "Photonmatter". We acknowledge useful discussions with A. Albus and F. Illuminati.
[1] A. G. Truscott, K. E. Strecker, W. I. McAlexander, G. B. Patridge, and R. G. Hulet, Science 291, 2570 (2001).

[2] F. Schreck, L. Khaykovich,K. L. Corwin, G. Ferrari, T. Bourdel, J. Cubizolles, and C. Salomon, Phys. Rev. Lett. 87, 080403 (2001).

[3] Z. Hadzibabic, C. A. Stan, K. Dieckmann, S. Gupta, M. W. Zwierlein, A. Gorlitz, and W. Ketterle, Phys. Rev. Lett. 88, 160401 (2002).

[4] G. Roati, F. Riboli, G. Modugno, M. Inguscio, Phys. Rev. Lett. 89, 150403 (2002).

[5] K. Molmer, Phys. Rev. Lett. 80, 1804 (1998).

[6] R. Roth, Phys. Rev. A 66, 013614 (2002).

[7] T. Miyakawa, T. Suzuki, and H. Yabu, Phys. Rev. A 64, 033611 (2001).

[8] H. Heiselberg, C. J. Pethick, H. Smith, and L. Viverit, Phys. Rev. Lett. 85, 2418 (2000); M. J. Bijlsma, B. A. Heringa, and H. T. C. Stoof, Phys. Rev. A 61, 053601 (2000).
[9] T. Miyakawa, T. Suzuki, and H. Yabu, Phys. Rev. A 62, 063613 (2000).

[10] X.-J. Liu and H. Hu, cond-mat/0212169

[11] H. Hu, X.-J. Liu, and M. Modugno, cond-mat/0301182 to appear on Phys. Rev. A (2003).

[12] G. Modugno, G. Roati, F. Riboli, F. Ferlaino, R. J. Brecha, and M. Inguscio, Science 297, 2240 (2002).

[13] A. Simoni, F. Ferlaino, G. Roati, G. Modugno, M. Inguscio, Phys. Rev. Lett. 90, 163202 (2003).

[14] G. Modugno, M. Modugno, F. Riboli, G. Roati, M. Inguscio, Phys. Rev. Lett. 89, 190404 (2002); F. Riboli and M. Modugno, Phys. Rev. A 65, 063614 (2002).

[15] E. G. M. van Kempen, S. J. J. M. F. Kokkelmans, D. J. Heinzen, and B. J. Verhaar, Phys. Rev. Lett. 88, 093201 (2002).

[16] R. Roth and H. Feldmeier, Phys. Rev. A 65, 021603(R) (2002).

[17] Y. Kagan, A. E. Muryshev, and G. V. Shlyapnikov, Phys. 
Rev. Lett. 81, 933 (1998).

[18] C. A. Sackett, H. T. C. Stoof, and R. G. Hulet, Phys. Rev. Lett. 80, 2031 (1998).

[19] Those two points represent the last stable samples along to two evaporation routes that led to the collapse, in the series of measurements described in [12].

[20] A. P. Albus, F. Illuminati and M. Wilkens, cond-mat/0211060
[21] C. C. Bradley, C. A. Sackett, and R. G. Hulet, Phys. Rev. Lett. 78, 985 (1997).

[22] F. Dalfovo, S. Giorgini, L. P. Pitaevskii, S. Stringari, Rev. Mod. Phys. 71, 463 (1999).

[23] See C. M. Savage, N. P. Robins, and J. J. Hope, Phys. Rev. A 67, 014304 (2003), and references therein. 